\title{
Higher inter-hemispheric homotopic connectivity in lifelong premature ejaculation patients: a pilot resting-state fMRI study
}

\author{
Nana Feng ${ }^{1,2 \#}$, Ming Gao ${ }^{3,4 \#}$, Jiayu Wu ${ }^{1,2}$, Guang Yang ${ }^{1,2}$, Ruiqing Piao ${ }^{1,2}$, Peng Liu ${ }^{1,2}$ \\ ${ }^{1}$ Life Science Research Center, School of Life Science and Technology, Xidian University, Xi'an, China; ${ }^{2}$ Engineering Research Center of Molecular \\ and Neuro Imaging Ministry of Education, School of Life Science and Technology, Xidian University, Xi'an, China; ${ }^{3}$ Xi'an Daxing Hospital of \\ Shaanxi University of Chinese Medicine, Xi'an, China; ${ }^{4}$ Department of Andrology, Xiyuan Hospital, China Academy of Chinese Medical Sciences, \\ Beijing, China
}

\#These authors contributed equally to this work.

Correspondence to: Peng Liu. Life Sciences Research Center, School of Life Science and Technology, Xidian University, Xi'an 710071, China. Email: liupengphd@gmail.com.

Background: Lifelong premature ejaculation (PE) is one common male sexual dysfunction and is implicated in widespread structural and functional abnormalities of bilateral hemispheres. However, whether the inter-hemisphere functional connectivity (FC) of lifelong PE patients was altered still remain unclear.

Methods: Thirty-four lifelong PE patients and 30 healthy controls (HCs) were enrolled in this study and all underwent T1-weighted and resting-state functional MRI (fMRI) scan. The voxel-mirrored homotopic connectivity (VMHC) measure and independent sample $t$-test were applied to examine the alterations of VMHC values in the patients relative to $\mathrm{HCs}$ with the significant threshold at $\mathrm{P}<0.05$, false discovery rates corrected. Correlation analysis was adopted to calculate the relationships between the imaging results and clinical characteristics of patients $(\mathrm{P}<0.05$, Bonferroni corrected). Receiver operating characteristic (ROC) curve analysis was performed to investigate the possible biomarkers for distinguishing the patients from the HCs using the VMHC values of inter-group differences.

Results: The results revealed that compared with HCs, lifelong PE patients had higher VMHC values in the precentral gyrus (PG), primary somatosensory cortex (S1), supplementary motor area (SMA), precuneus, middle temporal cortex (MTC), superior temporal pole (STP), thalamus, caudate and middle cingulate cortex (MCC). Correlation analysis showed that the mean VMHC values in the S1 negatively correlated with intravaginal ejaculation latency time (IELT) in the patient group. Furthermore, the caudate revealed the well classification power from the ROC analysis.

Conclusions: The present study showed the abnormal inter-hemisphere interaction and integration of information involved in ejaculation inhibitory control, sensorimotor mediation and self-reference processing including the thalamus, caudate, MCC, widespread parietal cortex and temporal cortex in lifelong PE patients compared with HCs. Correlation analysis and ROC analysis revealed the importance of S1 and caudate in lifelong PE. Notably, the ROC result of caudate might show the core roles of caudate played in the pathophysiology of lifelong PE.

Keywords: Lifelong premature ejaculation (lifelong PE); resting-state functional MRI (resting-state fMRI); functional connectivity (FC); voxel-mirrored homotopic connectivity (VMHC)

Submitted Sep 29, 2020. Accepted for publication Mar 23, 2021.

doi: 10.21037 /qims-20-1103

View this article at: http://dx.doi.org/10.21037/qims-20-1103 


\section{Introduction}

Lifelong premature ejaculation (PE) is one common male ejaculation dysfunction disease, which affected 3\% Chinese general male population (1) and seriously affected the life happiness of PE patients (2). It is defined as the ejaculation that always or nearly always happens within 1 minute of vaginal penetration as well as the insufficient ejaculation control (3). Substantial studies have reported the associations between the ejaculation and central nervous system (CNS) $(4,5)$, indicating the important roles that neural mechanisms underlying lifelong $\mathrm{PE}$ such as abnormal central serotonergic neurotransmission plays in its pathology and related treatment (6). Meanwhile, studies have shown the effects of genetic (7), prenatal androgen (8) and psychology (9) on the occurring of lifelong PE. However, the exact pathophysiological mechanisms are still ambiguous.

Recently, the neuroimaging studies has investigated the structural and functional alterations in lifelong PE (or PE) patients, including (I) the anatomical abnormalities in the superior frontal gyrus, precentral gyrus (PG) and limbic system (the left anterior cingulate cortex and bilateral posterior cingulate cortex) (10) as well as caudate nucleus (11); (II) anomalous resting-state brain activity in the inferior frontal gyrus (IFG) and functional activation in the middle temporal cortex (MTC) associated with sexual response (12); aberrant brain current activity in the parahippocampal gyrus (13) and inferior occipital gyrus (14) related to erotic stimuli and sertraline treatment; (III) abnormal functional connectivity (FC) involved in the IFG (15), the MTC (12) and medial orbital frontal cortex (16), supplementary motor area (SMA) (17), insula $(12,17)$, anterior and middle cingulate cortex (MCC), hypothalamus (18), caudate and putamen (19); (IV) and altered network characteristics, such as increased node degree in the right IFG and right MCC (17) as well as degree centrality (DC) in the insula and orbitofrontal cortex (20). Therefore, the aforementioned studies may suggest the central neural abnormalities, which may be implicated in the physiopathology of lifelong PE. However, the neuroimaging studies on the CNS of lifelong PE patients remain uncomplete.

Voxel-mirrored homotopic connectivity (VMHC) (21) is a new measure based on resting-state functional MRI ( $\mathrm{fMRI}$ ) and is proposed to examine the degree of functional homotopy $(22,23)$ between the brain regions of geometric correspondence located on the bilateral hemispheres. Meanwhile, the functional homotopy has been suggested to be a critical characteristic of brain intrinsic connectivity architecture (23) and VMHC is devised to test the aberrant pattern of inter-hemisphere intrinsic connectivity by calculating the correlations between the resting-state fMRI recorded blood oxygen level dependent signals $(21,24)$ of homotopic regions. The differences of VMHC values between different bilateral homotopic regions are considered to reveal the different characteristics of interhemisphere information interaction involved in sensation and motor coordination (23). VMHC has been regarded as a reliable approaches and has been applied to several diseases to characterize the abnormal homotopic intrinsic FC that could underly the pathological mechanisms, such as autism (25), Parkinson's disease (26) and traumatic axonal injury (27). The corpus callosum is the largest fiber tract connecting the two cerebral hemispheres and coordinates the information from the both hemispheres (28). The latest structural imaging research of lifelong PE has reported outward shape expansions in the bilateral thalamus (29), which is supposed to play important roles in the etiology of lifelong PE (30) and has cortex connectivity with the corpus callosum (31). Meanwhile, the lifelong PE patients have revealed multiply structural and functional abnormalities in the widespread bilateral brain regions $(10,16,17,20)$. These raised a question: whether the whole-brain interhemisphere intrinsic FC of lifelong PE patients is altered?

Therefore, the aims of this study were to investigate the differences of functional coordination between the two hemispheres in lifelong PE patients and to examine whether there were significant correlations between imaging results and clinical characteristics. In this study, we hypothesized that the inter-hemisphere FC revealed by aforementioned VMHC values would be altered in lifelong PE patients compared with healthy controls (HCs) and the resultant VMHC values would correlate clinically.

\section{Methods}

\section{Participants}

Thirty-four lifelong PE patients and $30 \mathrm{HCs}$ were included in this study. Physical examination was applied to every participant and all participants did not receive any treatment of PE. The exclusion criteria for all participants comprised: (I) having neurological or psychiatric disease; (II) having a history of alcohol, nicotine or medicine abuse; (III) having trauma; and (IV) having any contraindication of MRI scanning. The inclusion criteria for patients comprised: (I) heterosexual males (32); (II) the average of intravaginal 
ejaculation latency time (IELT) over past 2 weeks $<1$ minute, measured by female sexual partner using stopwatch; (III) Premature Ejaculation Diagnostic Tool (PEDT) score $>11(33,34)$; (IV) International Index of Erectile Function-5 (IIEF-5) score $>21$ (35). The inclusion criteria for HCs comprised: (I) heterosexual males (32); (II) PEDT score <5; (III) IIEF-5 score > 21; (IV) age- and hand dominate-matched with patient group. Furthermore, SelfRating Anxiety Scale (SAS) and Self-Rating Depression Scale (SDS) were adopted to investigate their anxiety and depression level $(36,37)$.

\section{Image data acquisition}

All participants' imaging data were acquired based on a 3.0T GE MRI system (EXCITE, Milwaukee, WC, USA) at the local hospital. A standard 8 channel birdcage head coil was adopted and foam pads were used to restrain head motion. The high resolution T1 image data were acquired using the $3 \mathrm{D}$ spoiled gradient echo with the parameters set as follows: field of view $(\mathrm{FOV})=256 \times 256 \mathrm{~mm}^{2}$; slice thickness $1 \mathrm{~mm}$; data matrix $=256 \times 256$; repetition time $(\mathrm{TR})$ $=8.2 \mathrm{~ms}$, echo time $(\mathrm{TE})=3.2 \mathrm{~ms}$; flip angle $(\mathrm{FA})=12^{\circ}$; in plane resolution $=1 \times 1 \mathrm{~mm}^{2}$. The resting-state $\mathrm{fMRI}$ image data were acquired using the single-shot gradient echo with the parameters set as follows: FOV $=240 \times 240 \mathrm{~mm}^{2}$; slice thickness $3.5 \mathrm{~mm}$ with no gaps; matrix size $=64 \times 64$; $\mathrm{TR}=2,000 \mathrm{~ms}$, TE $=30 \mathrm{~ms} ; \mathrm{FA}=90^{\circ}$; in-plane resolution $=3.75 \times 3.75 \mathrm{~mm}^{2} ; 45$ axial slices. During scanning, each participant was required to keep in the supine position and think about nothing during the resting imaging.

\section{MRI data preprocessing}

The preprocessing of fMRI data were performed using DPABI (V4.1, http://rfmri.org/dpabi) (38) and Statistical Parametric Mapping 12 (spm12, http://www.fil.ion.ucl. ac.uk/spm/software/spm12/) software package based on the platform of MATLAB. Briefly, the following steps were conducted: (I) removal of first 5 time points; (II) correction of slice timing; (III) correction of head motion using rigid body transformation; (IV) spatial normalization to the MNI space using the standard echo planar imaging template and resampling the images at $3 \times 3 \times 3 \mathrm{~mm}^{3}$ voxel size (39); (V) smoothing with a Gaussian kernel $(6 \mathrm{~mm}$ full-width at half maximum); (VI) removal of linear detrending and bandpass filtering $(0.01-0.1 \mathrm{~Hz})$; (VII) nuisance covariates regression including 24 head motion parameters and the signals of white matter, cerebrospinal fluid signals and global brain. After motion correction, all participants enrolled in the following data processing had shorter than $2 \mathrm{~mm}$ translation in the $\mathrm{X}, \mathrm{Y}$ or $\mathrm{Z}$-axis or $2^{\circ}$ of angular rotation. Furthermore, the framewise displacement (FD) (40) of the two groups were examined and showed no inter-group differences in mean head motion.

\section{VMHC analysis}

VMHC measure supposed that the bilateral hemispheres had symmetrical morphology. The normalized T1 images of all participants were averaged and the resultant mean T1 image was further averaged with its left-right mirrored version to create the group-specific identical structural template. The above preprocessed functional images were then transformed to register to the new identical template. The VMHC calculation was conducted with software DPABI (38). The VMHC was computed as the Pearson correlations between pairs of symmetrical voxels of the two hemispheres, after which Fisher r-to-z transformation was adopted to improve the normality of the correlation coefficients and to generate the final VMHC map for each participant.

\section{Statistical analysis}

Statistical Product and Service Solutions (SPSS) version 20.0 (IBM Corporation, Armonk, New York, NY, USA) was applied to examine the inter-group differences of the key clinical characteristics (age, IELT, PEDT score, IIEF5 score, SAS and SDS) using two-tailed two sample $t$-test. The significant level was set as $\mathrm{P}<0.05$.

To test the differences of VMHC between the two groups, one sample and two sample $t$-test were used to restrict the imaging results to the brain regions with positive VMHC (41) and the inter-group contrasts adopted the threshold as $\mathrm{P}<0.05$ (FDR corrected with the age, anxiety, depression factors as covariates). The brain regions showing significant differences from above $t$-tests were supposed as regions of interest (ROIs). Correlation analysis with and without emotional factors (e.g., SAS and SDS) as covariates was used to test the association between the mean VMHC values of the ROIs and clinical characteristics, such as PEDT scores and IELT $(\mathrm{P}<0.05$, Bonferroni corrected). Furthermore, receiver operating characteristic (ROC) curves analysis (42) was performed using the mean VMHC values from the ROIs to figure out the possible biomarkers for separating the patients from HCs, including 
the characteristics of sensitivity, specificity and area under the curve (AUC) (43). Nonparametric permutation test was used to examine the statistical significance and $\mathrm{P}<0.05$ was considered to be significant in the ROC analysis.

\section{Results}

\section{Clinical characteristics results}

The statistical results of demographic and clinical characteristics of all the participants were shown in Table 1 .

Table 1 Demographic and clinical characteristics of all participants

\begin{tabular}{lccc}
\hline Characteristics & $P E(n=34)$ & HCs $(n=30)$ & $P$ value \\
\hline Ages (years) & $31.18 \pm 5.21$ & $31.70 \pm 2.91$ & 0.628 \\
IELT (min) & $0.64 \pm 0.23$ & $11.00 \pm 5.55$ & $0.000^{\star \star \star}$ \\
PEDT score & $17.06 \pm 1.71$ & $0.73 \pm 1.44$ & $0.000^{\star \star \star}$ \\
IIEF-5 score & $23.97 \pm 0.87$ & $24.27 \pm 1.34$ & 0.292 \\
SAS & $38.88 \pm 4.06$ & $30.47 \pm 1.68$ & $0.000^{\star \star \star}$ \\
SDS & $40.97 \pm 3.44$ & $30.50 \pm 2.13$ & $0.000^{\star \star \star}$ \\
\hline
\end{tabular}

Data were expressed as the mean $\pm \mathrm{SD}$. ${ }^{* *}, \mathrm{P}<0.001$ by independent sample $t$-test. PE, premature ejaculation; HCs, healthy controls; IELT, intravaginal ejaculatory latency time; PEDT, Premature Ejaculation Diagnostic Tool; IIEF, International Index of Erectile Function; SAS, Self-Rating Anxiety Scale; SDS, Self-Rating Depression Scale; SD, standard deviation.
No significant inter-group differences were observed in age and IIEF-5 scores (all P>0.05). The IELT, SAS and SDS scores significantly differed between the two groups (all $\mathrm{P}<0.001)$.

\section{Inter-group VMHC comparison}

Compared with HCs, lifelong PE patients showed significantly higher $\mathrm{VMHC}$ values in the $\mathrm{PG}$, primary somatosensory cortex (S1), SMA, precuneus, MTC, superior temporal pole (STP), thalamus, caudate and MCC (Figure 1 and Table 2).

\section{Correlation analysis}

Based on the inter-group VMHC results, correlation analysis showed that the mean $\mathrm{VMHC}$ values in the S1 negatively correlated with IELT $(\mathrm{r}=-0.38, \mathrm{P}=0.026$, uncorrected) (Figure $2 A$ ) in the patient group. After accounting for the emotional factors, the aforementioned significant correlation was still observed $(\mathrm{r}=-0.47, \mathrm{P}=0.005$, Bonferroni corrected) (Figure 2B). Furthermore, the ROC analysis revealed the AUC in caudate was $81.7 \%$ with a specificity of $87.2 \%$ and a sensitivity of $65.3 \%$ (Figure 3).

\section{Discussion}

Compared with HCs, we investigated the altered VMHC

Table 2 Brian regions with higher differences in VMHC in lifelong PE patients compared with HCs

\begin{tabular}{|c|c|c|c|c|c|c|}
\hline Brain regions & \multicolumn{3}{|c|}{$\mathrm{MNI}$} & BA & Cluster size & $\mathrm{T}$ value \\
\hline Precuneus & $15 /-15$ & -42 & 72 & 1,5 & 221 & 4.37 \\
\hline S1 & $18 /-18$ & -39 & 75 & $1,2,3$ & 677 & 4.19 \\
\hline Thalamus & $15 /-15$ & -15 & 12 & - & 223 & 4.09 \\
\hline STP & $48 /-48$ & 15 & -21 & 38 & 105 & 3.57 \\
\hline$P G$ & $24 /-24$ & -9 & 66 & 6 & 273 & 3.54 \\
\hline MTC & $54 /-54$ & -24 & -3 & 21 & 436 & 3.50 \\
\hline Caudate & $15 /-15$ & 9 & 21 & - & 153 & 3.48 \\
\hline
\end{tabular}

VMHC, voxel-mirrored homotopic connectivity; PE, premature ejaculation; HCs, healthy controls; MNI, Montreal Neurological Institute; $\mathrm{BA}$, Brodmann area; $(\mathrm{X}, \mathrm{Y}, \mathrm{Z})$, coordinate of peak location in the MNI space; T, statistical value of peak voxel; S1, primary somatosensory cortex; SMA, supplementary motor area; STP, superior temporal pole; PG, precentral gyrus; MTC, middle temporal cortex; MCC, middle cingulate cortex. 


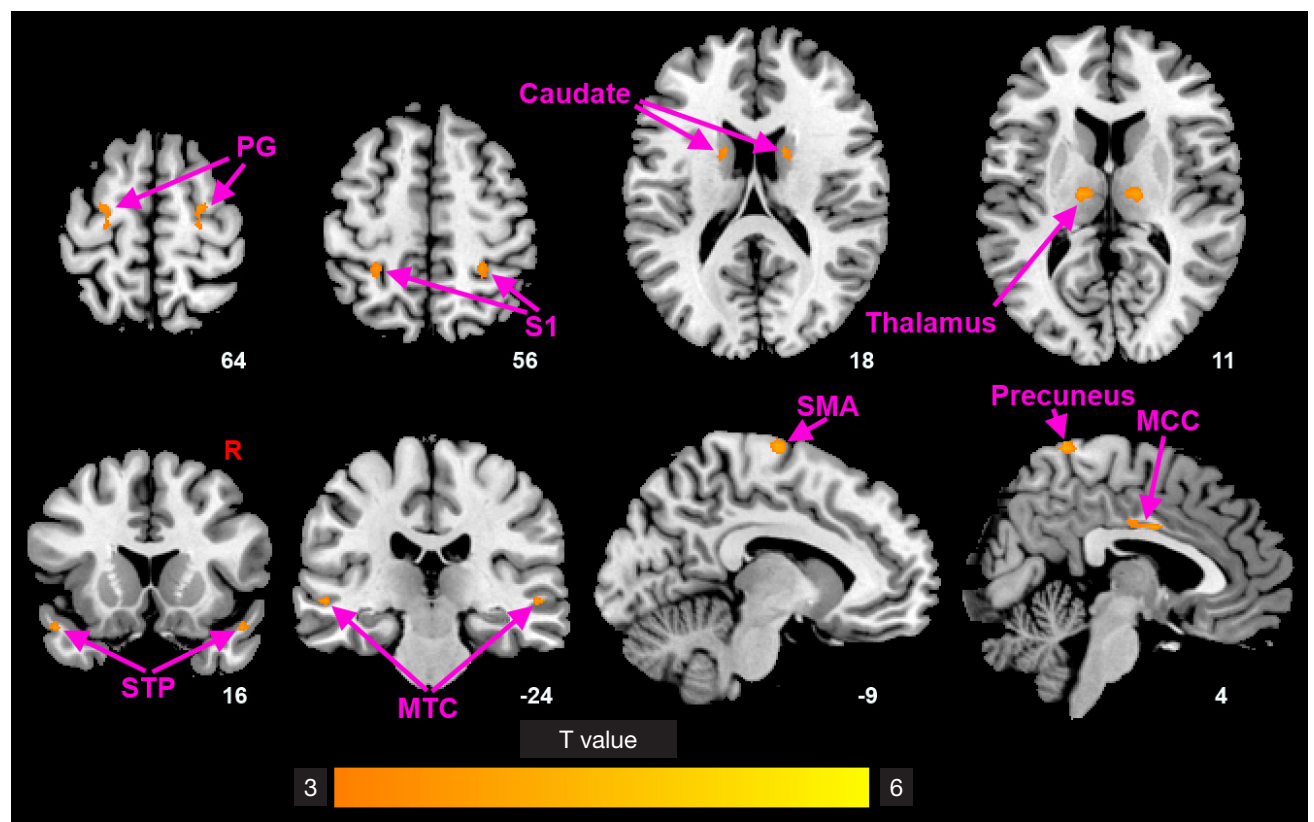

Figure 1 Brain regions showing significantly altered VMHC values in the lifelong PE patients compared with HCs. VMHC, voxel-mirrored homotopic connectivity; PE, premature ejaculation; HCs, healthy controls; PG, precentral gyrus; S1, primary somatosensory cortex; STP, superior temporal pole; MTC, middle temporal cortex; SMA, supplementary motor area; MCC, middle cingulate cortex; R, right.
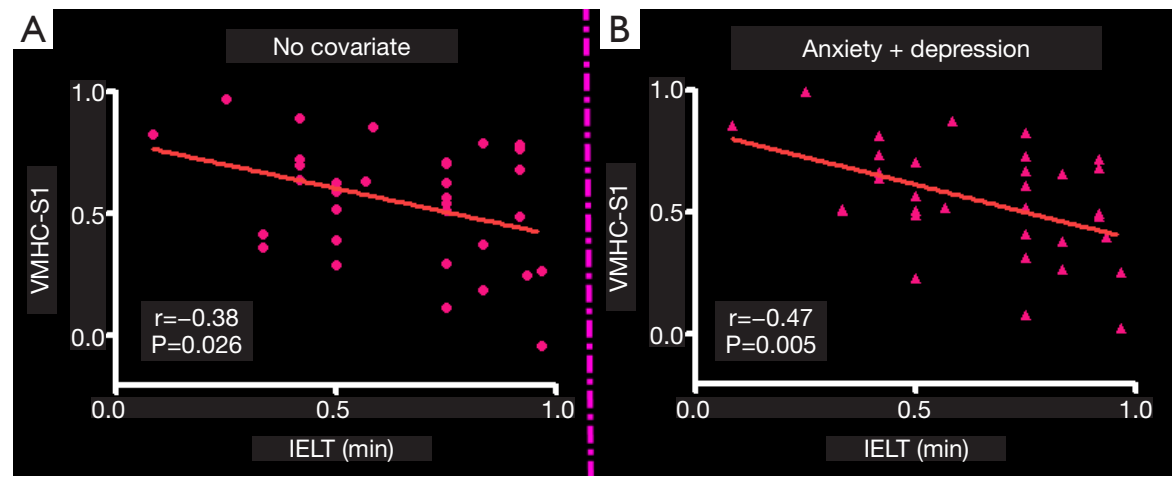

Figure 2 Correlations results. (A) Positive correlation between the VMHC values in the S1 and IELT in the patient group (with no covariate). (B) Positive correlation between the VMHC values in the S1 and IELT in the patient group (with the anxiety and depression scores as covariates). VMHC, voxel-mirrored homotopic connectivity; S1, primary somatosensory cortex; IELT, intravaginal ejaculatory latency time.

of lifelong PE patients using the VMHC measure and the inter-group statistical analysis results showed that the higher VMHC values were found in the PG, S1, SMA, precuneus, MTC, STP, thalamus, caudate and MCC than in the HCs. The mean VMHC values of S1 had significant correlation with IELT and the VMHC values of caudate revealed well power to differentiate lifelong PE patients from the HCs.

In this study, the higher VMHC values in temporal regions including the MTC and the STP were found in lifelong PE patients than in the HCs. Male sexual behavior is reported as a complex process involved in the excitation, erection and the ejaculation stage. Ejaculation is the culmination of sexual behavior and mediated by special regions implicated in sensory or sexual inhibition (15). Healthy males showed increased brain activity of temporal cortex after ejaculation (44). The temporal cortex is associated with visual information and 


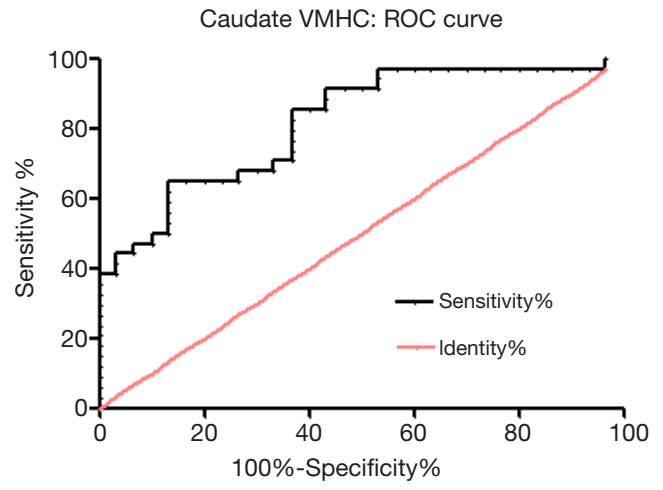

Figure 3 ROC curve analysis of the mean VMHC values of caudate. The AUC for caudate was $81.7 \%$ with a specificity of $87.2 \%$ and a sensitivity of $65.3 \%$. ROC, receiver operating characteristic; VMHC, voxel-mirrored homotopic connectivity; AUC, area under the curve.

autonomic processing (45). Numerous studies have revealed the dysfunction of temporal cortex in PE patients (12,16-19). For example, a recent $\mathrm{PE}$ study has reported the increased activation in right MTC during sexual picture stimuli compared with scenery picture stimuli (12). Meanwhile, decreased current source density (CSD) in the left MTC (13) was found in another PE studies during sexual video stimuli using HCs as baseline. Therefore, the temporal cortex dysfunction might be important in lifelong PE and be mainly involved in the abnormalities of ejaculation inhibitory control. Furthermore, the recent lifelong PE study showed the increased right MTC-seeded FC in the multiple regions including the left MTC compared with HCs (46), which was consistent with our imaging results found here.

We observed that lifelong PE patients had higher VMHC values in the S1, thalamus, SMA, PG and MCC in this study. Ejaculation is a motivated process and the alteration of penis sensitivity is an important feature of PE. The abnormalities of genital somatosensory information processing are highly involved in sexual dysfunction (47). Thalamus is the crucial region associated with homeostatic, motivational and visceral sensory response (48), which is also involved in the androgen receptor expression and participants in the spinal cord control of ejaculation (49). Neuroimaging study has revealed that the sensory information output from the penis is transmitted to the spinal ejaculation center, the thalamus and finally to the S1 (50). Healthy males showed increased activation in somatosensory cortices by viewing sexual scenes (51).
Lifelong PE patients had decreased FC density in thalamus (17) as well as decreased FC involved in S1 (16). Furthermore, genital stimuli induced increased brain activation in sensory and motor cortex including the S1 and premotor cortex in HCs (52). Meanwhile, a recent study reported the increased excitability of motor cortex whether in heterosexual or homosexual healthy males (53) by visual sexual stimuli, which revealed the participation of motor except aforementioned sensory in sexual behavior. The SMA and PG are typical motor association cortex. Lifelong PE patients showed abnormal increased thickness in bilateral PG (10) and increased long-range FC density in bilateral SMA as well (12) as increased right MTC-seed $\mathrm{FC}$ in bilateral SMA in lifelong PE patients compared with HCs (17). The cingulate cortex participants in sexual erection process (54) and the MCC is involved in sensorimotor processing revealed by its strongly connectivity with regions related to sensory and motor, such as insula and SMA (55). The functional abnormalities of bilateral MCC have been found in lifelong PE patients $(12,17)$. Therefore, studies mentioned above may indicate the important roles that $\mathrm{S} 1$ and thalamus play in sensory processing as well as SMA and PG play in motor preparation processing of lifelong $\mathrm{PE}$ pathology besides the MCC in sensorimotor. The greater VMHC values found in S1, thalamus, SMA, PG and MCC might represent the abnormal inter-hemisphere functional interaction and integration associated with sensorimotor processing and might compensate for other brain functions. Furthermore, the mean VMHC values extracted from the S1 were negatively correlated with the IELT in the patient group with and without accounting for emotional factors, which might highlight the special roles of S1 plays in the pathology of lifelong PE.

In this study, lifelong PE patients showed higher VMHC in the precuneus, caudate compared with HCs. Parietal cortex is mainly involved in somatosensory and visualspatial processing (56). Specially, precuneus is located in the posteromedial parietal cortex and is associated with the visual-spatial perception as well as self-consciousness and self-related mental processing (57). Healthy males had increased regional cerebral brain flow (rCBF) in the right precuneus during ejaculation relative to sexual stimuli (5). Lifelong PE patients showed increased DC in the bilateral precuneus compared with HCs (20). Caudate is the important part of striatum and play roles in sex-related reward and motor-mediation processing (58). Structural study of lifelong PE patients found larger mean volume 
in caudate (11) in the patient group compared with HCs. Meanwhile, lifelong PE patients had deceased FC between the right caudate and multiple regions involved in abnormal sensory and reward processing using HCs as baseline (19). In addition, lifelong PE patients revealed increased longrange $\mathrm{FC}$ density in the bilateral precuneus compared with HCs as well as decreased short-range FC density in the left caudate (17). Therefore, the higher VMHC values found in precuneus and caudate might indicate the inter-hemisphere functional abnormalities involved in the self-reference information processing of sexual stimuli of lifelong PE. Furthermore, the ROC analysis showed that the caudate of the lifelong PE patients and HCs had the quite well performance to distinguish the patients from HCs with the AUC of $81.7 \%$, indicating the VMHC values from the caudate could be potential biomarkers for lifelong PE and suggesting the core participation of caudate dysfunction in the pathophysiological process of lifelong PE.

\section{Limitations}

There are several limitations in this study. First, the results of the current findings were generated from a relatively small sample data of the patients and HCs. Second, whether the VMHC abnormality found in this study was the cause or the outcome of lifelong PE was still unclear. Third, this study is a cross sectional study, the dynamic process of VMHC alterations was unbale to be observed. Furthermore, this study was not applied principal component and much descriptive statistics analyses, which might improve the validation of the original presumptions (59). Thereby, further studies with larger number of participants and longitudinal data are needed to validate and perfect our findings as well as the application of analyses related to component and multivariate statistics.

\section{Conclusions}

Our present study provided evidences that patients with lifelong PE had the abnormal inter-hemisphere interaction and integration of information involved in ejaculation inhibitory control, sensorimotor mediation and selfreference processing in widespread cortex (e.g., MTC, STP, S1, SMA and PG) and subcortex regions (e.g., thalamus, caudate and MCC) compared with HCs. The statistical results of the correlation and ROC analysis using imaging findings might indicate the importance of $\mathrm{S} 1$ and caudate in lifelong PE. Especially the result from the ROC analysis might explore the core roles of caudate played in the pathophysiology of lifelong PE.

\section{Acknowledgments}

Funding: This study was financially supported by the National Natural Science Foundation of China (Nos. 81771918, 62001370, 61971425), Shaanxi National Science Foundation (No. 2020JM-197), China Postdoctoral Science Foundation Grant (No. 2019M650985), Development Funds of Shaanxi Science and Technology Agency of China (No. 2018SF/091).

\section{Footnote}

Conflicts of Interest: All authors have completed the ICMJE uniform disclosure form (available at http://dx.doi. org/10.21037/qims-20-1103). The authors have no conflicts of interest to declare.

Ethical Statement: The ethical committee of the Northwest Women's and Children's Hospital in China approved the all procedures of this study (No. 2017014), and which was in accordance with the Code of Ethics of the World Medical Association (Declaration of Helsinki). All participants provided the written informed consents before the experiment began.

Open Access Statement: This is an Open Access article distributed in accordance with the Creative Commons Attribution-NonCommercial-NoDerivs 4.0 International License (CC BY-NC-ND 4.0), which permits the noncommercial replication and distribution of the article with the strict proviso that no changes or edits are made and the original work is properly cited (including links to both the formal publication through the relevant DOI and the license). See: https://creativecommons.org/licenses/by-nc-nd/4.0/.

\section{References}

1. Gao J, Zhang X, Su P, Liu J, Xia L, Yang J, Shi K, Tang D, Hao Z, Zhou J. Prevalence and factors associated with the complaint of premature ejaculation and the four premature ejaculation syndromes: a large observational study in China. J Sex Med 2013;10:1874-81.

2. Althof SE, Abdo CHN, Dean J, Hackett G, Mccabe MP, Mcmahon CG, Rosen RC, Sadovsky R, Waldinger MD, Becher E. International society for sexual medicine's 
guidelines for the diagnosis and treatment of premature ejaculation. J Sex Med 2010;7:2947-69.

3. McMahon CG, Althof SE, Waldinger MD, Porst H, Dean J, Sharlip ID, Adaikan PG, Becher E, Broderick GA, Buvat J, Dabees K, Giraldi A, Giuliano F, Hellstrom WJG, Incrocci L, Laan E, Meuleman E, Perelman MA, Rosen RC, Rowland DL, Segraves R. An evidence-based definition of lifelong premature ejaculation: report of the international society for sexual medicine (ISSM) ad hoc committee for the definition of premature ejaculation. J Sex Med 2008;5:1590-606.

4. Holstege G. Central nervous system control of ejaculation. World J Urol 2005;23:109-14.

5. Holstege G, Georgiadis JR, Paans AMJ, Meiners LC, Reinders AATS. Brain activation during human male ejaculation. J Neurosci 2003;23:9185-93.

6. Waldinger MD. The neurobiological approach to premature ejaculation. J Urol 2002;168:2359-67.

7. Janssen PK, Zwinderman AH, Olivier B, Waldinger MD. Serotonin transporter promoter region (5-HTTLPR) polymorphism is not associated with paroxetine-induced ejaculation delay in Dutch men with lifelong premature ejaculation. Korean J Urol 2014;55:129-33.

8. Bolat D, Kocabas G, Kose T, Degirmenci T, Aydin M, Dincel C. The relationship between the second to fourth digit ratios and lifelong premature ejaculation: a prospective, comparative study. Andrology 2017;5:535-40.

9. McCabe M, Althof SE, Assalian P, Chevret-Measson M, Leiblum SR, Simonelli C, Wylie K. Psychological and interpersonal dimensions of sexual function and dysfunction. J Sex Med 2010;7:327-36.

10. Guo F, Xi YB, Gao M, Liu L, Fei NB, Qin W, Li C, Cui LB, Yan F, Yu L. Alterations in cortical thickness in nonmedicated premature ejaculation patients: a morphometric MRI study. J Magn Reson Imaging 2018;47:656-62.

11. Atalay HA, Sonkaya AR, Ozbir S, Culha MG, Canat L. Are there differences in brain morphology in patients with lifelong premature ejaculation? J Sex Med 2019;16:992-8.

12. Zhang B, Lu J, Xia J, Wang F, Li W, Chen F, Han Y, Chen Y, Zhu B, Qing Z. Functional insights into aberrant brain responses and integration in patients with lifelong premature ejaculation. Sci Rep 2017;7:460.

13. Hyun JS, Kam SC, Kwon O. Changes of cerebral current source by audiovisual erotic stimuli in premature ejaculation patients. J Sex Med 2008;5:1474-81.

14. Kwon OY, Kam SC, Choi JH, Do JM, Hyun JS. Effects of sertraline on brain current source of the high beta frequency band: analysis of electroencephalography during audiovisual erotic stimulation in males with premature ejaculation. Int J Impot Res 2011;23:213-9.

15. Yang X, Gao M, Zhang L, Liu L, Liu P, Sun J, Xi Y, Yin H, Qin W. Central neural correlates during inhibitory control in lifelong premature ejaculation patients. Front Hum Neurosci 2018;12:206.

16. Xu Z, Yang X, Gao M, Liu L, Sun J, Liu P, Qin W. Abnormal resting-state functional connectivity in the whole brain in lifelong premature ejaculation patients based on machine learning approach. Front Neurosci 2019;13:448.

17. Lu J, Zhang X, Wang H, Qing Z, Han P, Li M, Xia J, Chen F, Yang B, Zhu B. Short- and long-range synergism disorders in lifelong premature ejaculation evaluated using the functional connectivity density and network property. Neuroimage Clin 2018;19:607-15.

18. Gao M, Feng N, Wu J, Sun J, Zhang L, Guo X, Yuan J, Guo J, Liu P. Altered functional connectivity of hypothalamus in lifelong premature ejaculation patients. J Magn Reson Imaging 2020;52:778-84.

19. Gao M, Feng N, Guo B, Wu J, Liu P. Striatum-related intrinsic connectivity deficits in lifelong premature ejaculation patients. Urology 2020;143:159-64

20. Gao M, Feng N, Liu X, Sun J, Hou G, Zhang L, Yin H, Guo B, Wu J, Huang M, Yuan J, Guo J, Liu P. Abnormal degree centrality in lifelong premature ejaculation patients: an fMRI study. Brain Imaging Behav 2020. [Epub ahead of print]. doi: 10.1007/s11682-020-00340-4.

21. Zuo XN, Kelly C, Di Martino A, Mennes M, Margulies DS, Bangaru S, Grzadzinski R, Evans AC, Zang YF, Castellanos FX, Milham MP. Growing together and growing apart: regional and sex differences in the lifespan developmental trajectories of functional homotopy. J Neurosci 2010;30:15034-43.

22. Salvador R, Suckling J, Martin RC, John DP, Menon D. Neurophysiological architecture of functional magnetic resonance images of human brain. Cereb Cortex 2005;15:1332-42.

23. Stark DE, Margulies DS, Shehzad ZE, Reiss P, Kelly AM, Uddin LQ, Gee DG, Roy AK, Banich MT, Castellanos FX, Milham MP. Regional variation in interhemispheric coordination of intrinsic hemodynamic fluctuations. J Neurosci 2008;28:13754-64.

24. Fox MD, Raichle ME. Spontaneous fluctuations in brain activity observed with functional magnetic resonance imaging. Nat Rev Neurosci 2007;8:700-11.

25. Anderson JS, Druzgal TJ, Froehlich AL, Dubray MB, 
Lange N, Alexander AL, Abildskov TJ, Nielsen JA, Cariello AN, Cooperrider JR. Decreased interhemispheric functional connectivity in autism. Cereb Cortex 2011;21:1134-46.

26. Luo C, Guo X, Song W, Zhao B, Cao B, Yang J, Gong Q, Shang H. Decreased resting-state interhemispheric functional connectivity in Parkinson's disease. Biomed Res Int 2015;2015:692684.

27. Li J, Gao L, Xie K, Zhan J, Luo X, Wang H, Zhang H, Zhao J, Zhou F, Zeng X. Detection of functional homotopy in traumatic axonal injury. Eur Radiol 2017;27:325-35.

28. van der Knaap LJ, van der Ham IJ. How does the corpus callosum mediate interhemispheric transfer? A review. Behav Brain Res 2011;223:211-21.

29. Lu J, Yuan L, Jin J, Yang S, Zhang W, Li M, Zhang X, Wang J, Wu S, Chen Q. Brain cortical complexity and subcortical morphometrics in lifelong premature ejaculation. Front Hum Neurosci 2020;14:283.

30. Gao M, Yang X, Liu L, Fei N, Xi Y, Guo F, Yan F, Meng $\mathrm{P}, \mathrm{Yu}$ L, Liu P. Abnormal white matter microstructure in lifelong premature ejaculation patients identified by tractbased spatial statistical analysis. J Sex Med 2018;15:1272-9.

31. Jarbo K, Verstynen T, Schneider W. In vivo quantification of global connectivity in the human corpus callosum. Neuroimage 2012;59:1988-96.

32. Wang D, Han L, Xi C, Xu Y, Lai J, Lu S, Huang M, $\mathrm{Hu}$ J, Wei N, Xu W, Zhou W, Lu Q, He H, Hu S. Interactive effects of gender and sexual orientation on cortical thickness, surface area and gray matter volume: a structural brain MRI study. Quant Imaging Med Surg 2020;10:835-46.

33. Symonds T, Perelman MA, Althof SE, Giuliano F, Martin ML, May K, Abraham L, Crossland A, Morris M. Development and validation of a premature ejaculation diagnostic tool. Eur Urol 2007;52:565-73.

34. Huang YP, Chen B, Ping P, Wang HX, Hu K, Zhang T, Yang H, Jin Y, Yang Q, Huang YR. The premature ejaculation diagnostic tool (PEDT): linguistic validity of the Chinese version. J Sex Med 2014;11:2232-8.

35. Rosen RC, Cappelleri JC, Smith, Lipsky J, Pena BM. Development and evaluation of an abridged, 5-item version of the International Index of Erectile Function (IIEF-5) as a diagnostic tool for erectile dysfunction. Int J Impot Res 1999;11:319-26.

36. Zung WW. A rating instrument for anxiety disorders. Psychosomatics 1971;12:371-9.

37. Zung WW. A self-rating depression scale. Arch Gen
Psychiatry 1965;12:63-70.

38. Yan CG, Wang XD, Zuo XN, Zang YF. DPABI: Data Processing \& Analysis for (Resting-State) Brain Imaging. Neuroinformatics 2016;14:339-51.

39. Calhoun VD, Wager TD, Krishnan A, Rosch KS, Seymour KE, Nebel MB, Mostofsky SH, Nyalakanai P, Kiehl KA. The impact of T1 versus EPI spatial normalization templates for $\mathrm{fMRI}$ data analyses. Hum Brain Mapp 2017;38:5331-42.

40. Van Dijk KR, Sabuncu MR, Buckner RL. The influence of head motion on intrinsic functional connectivity MRI. Neuroimage 2012;59:431-8.

41. Murphy K, Birn RM, Handwerker DA, Jones TB, Bandettini PA. The impact of global signal regression on resting state correlations: are anti-correlated networks introduced? Neuroimage 2009;44:893-905.

42. Swets JA. ROC analysis applied to the evaluation of medical imaging techniques. Invest Radiol 1979;14:109-21.

43. Hanley JA, McNeil BJ. The meaning and use of the area under a receiver operating characteristic (ROC) curve. Radiology 1982;143:29-36.

44. Mallick HN, Tandon S, Jagannathan NR, Gulia KK, Kumar VM. Brain areas activated after ejaculation in healthy young human subjects. Indian J Physiol Pharmacol 2007;51:81-5.

45. Westerhaus MJ, Loewy AD. Central representation of the sympathetic nervous system in the cerebral cortex. Brain Res 2001;903:117-27.

46. Zhang T, Tang D, Cai H, Zhang B, Yu Y. Selective functional hyperconnectivity in the middle temporal gyrus subregions in lifelong premature ejaculation. J Sex Med 2020;17:1457-66.

47. Yang CC, Bowen JD, Kraft GH, Uchio EM, Kromm BG. Physiologic studies of male sexual dysfunction in multiple sclerosis. Mult Scler 2001;7:249-54.

48. Cechetto DF, Saper CB. Evidence for a viscerotopic sensory representation in the cortex and thalamus in the rat. J Comp Neurol 1987;262:27-45.

49. Swaab DF. Sexual differentiation of the brain and behavior. Best Pract Res Clin Endocrinol Metab 2007;21:431-44.

50. Veening JG, Coolen LM. Neural mechanisms of sexual behavior in the male rat: emphasis on ejaculation-related circuits. Pharmacol Biochem Behav 2014;121:170-83.

51. Ferretti A, Caulo M, Gratta CD, Matteo RD, Merla A, Montorsi F, Pizzella V, Pompa P, Rigatti P, Rossini PM. Dynamics of male sexual arousal: distinct components of brain activation revealed by fMRI. Neuroimage 2005;26:1086-96. 
52. Wise NJ, Frangos E, Komisaruk BR. Activation of sensory cortex by imagined genital stimulation: an fMRI analysis. Socioaffect Neurosci Psychol 2016;6:31481.

53. Schecklmann M, Engelhardt K, Konzok J, Rupprecht R, Greenlee MW, Mokros A, Langguth B, Poeppl TB. Sexual motivation is reflected by stimulus-dependent motor cortex excitability. Soc Cogn Affect Neurosci 2015;10:1061-5.

54. Steers WD. Neural pathways and central sites involved in penile erection: neuroanatomy and clinical implications. Neurosci Biobehav Rev 2000;24:507-16.

55. Yu C, Zhou Y, Liu Y, Jiang T, Dong H, Zhang Y, Walter M. Functional segregation of the human cingulate cortex is confirmed by functional connectivity based neuroanatomical parcellation. Neuroimage 2011;54:2571-81.

56. Dieterich M, Brandt T. The parietal lobe and the vestibular system. Handb Clin Neurol 2018;151:119-40.

57. Cavanna AE, Trimble MR. The precuneus: a review of its functional anatomy and behavioural correlates. Brain 2006;129:564-83.

58. Rodríguez-Manzo G, Pellicer F. Electrical stimulation of dorsal and ventral striatum differentially alters the copulatory behavior of male rats. Behav Neurosci 2010;124:686-94.

59. Pearson J, Kosslyn SM. The heterogeneity of mental representation: ending the imagery debate. Proc Natl Acad Sci U S A 2015;112:10089-92.
Cite this article as: Feng N, Gao M, Wu J, Yang G, Piao R, Liu P. Higher inter-hemispheric homotopic connectivity in lifelong premature ejaculation patients: a pilot resting-state fMRI study. Quant Imaging Med Surg 2021;11(7):3234-3243. doi: 10.21037/qims-20-1103 